\title{
Quality assurance monitoring of osteopathic manipulative treatment
}

\author{
RICHARD W. KOSS, DO
}

The quality assurance review program of military medicine has implications for civilian osteopathic medicine. Clinical monitors need to be developed that are based on the principles of osteopathy. Components of a quality assurance screening checklist for osteopathic manipulative treatment include credentials of the osteopathic physician, the diagnosis, either somatic dysfunction or other condition, the type of osteopathic manipulative treatment applied, and specific contraindications. A checklist applicable to osteopathic manipulative treatment that could be used by military and civilian sectors is suggested.

Quality assurance is the watchdog of the medical care delivery system. It is a rapidly growing program that covers all aspects of medicine. Military medicine has had a quality assurance review program for many years. It is complex and difficult to understand. Osteopathic manipulative treatment is now being placed in this military quality assurance process.

In February 1988, US Navy osteopathic physician, J. Klos, DO, sent a letter to all colleges of osteopathic medicine, the American Osteopathic Association, and the American Acad-

\footnotetext{
Dr Koss is assistant professor, Department of Osteopathic Manipulative Medicine, Kirksville College of Osteopathic Medicine, Kirksville, Mo.

Reprint requests to Richard W. Koss, DO, Department of Osteopathic Manipulative Medicine, Kirksville College of Osteopathic Medicine, 800 W Jefferson, Kirksville, MO 63501 .
}

emy of Osteopathy requesting information on the quality assurance monitoring of osteopathic manipulative treatment (OMT) methods used in civilian medical practice. $\mathrm{He}$ wished to use this information to establish clinical indications for the use of OMT, and to develop guidelines for the performance of osteopathic manipulation in the military.

There has been very little response from the civilian osteopathic community. The ramifications and implications for the osteopathic profession if this request is not addressed are enormous. An example of how to develop a quality assurance monitor to the satisfaction of the military may shed some insight into the entire quality assurance process and its current expansion into civilian medical practice. It is written from a background of 4 years' experience as an Air Force physician who developed quality assurance clinical monitors and reviewed many charts.

\section{Consequences of quality assurance monitoring}

In the military, all aspects of medicine-blood pressure measurements taken by a technician to the most intricate details of surgery-have come under close scrutiny. These procedures are reviewed and rereviewed according to criteria established by the military medical administration. The review process begins with a screening checklist of important information that must be documented on the chart. Any discrepancy, patient complaint, or bad outcome starts a cascade of committee reviews to determine the quality, appropriateness, and li- 
ability of the medical care rendered. This process has been referred to as the "fishbowl phenomenon."

Initiation of a quality assurance review previously was based on patient complaints. Osteopathic manipulative treatment has been relatively immune from this intense review process for several reasons. First, military osteopathic physicians performing OMT have had excellent results with no complaints or serious side effects. Osteopathic physicians have helped military personnel stay healthy without the use of drugs. There has been no need for quality assurance review, since there have not been patient complaints. Second, military allopathic physicians have little to no understanding of OMT. In the past, the right of DOs to perform OMT was based on graduation from an accredited college of osteopathic medicine. Now OMT will fall under the watchful eyes of quality assurance monitoring.

It is possible that OMT will be regulated, controlled, or even eliminated in military and civilian medicine and no longer will be available as a viable alternative. The governing bodies regulating military and civilian medicine, made up mostly of allopathic physicians, are not cognizant of the benefits of OMT. Procedures that are not understood as a standard of care by the regulating authority may be overly scrutinized and therefore criticized or even regulated into obscurity. Such scrutiny is exemplified by a recent instance of prohibition of OMT at a military facility. In September 1987, the Association of Military Osteopathic Physicians and Surgeons ${ }^{2}$ reported successful efforts to reverse a discriminatory ruling against OMT made at a naval facility. This incident shows that it is vital for the osteopathic profession to be aware of all discriminatory situations and act accordingly, otherwise more of this kind of situation may occur.

Why should the civilian sector spend time helping its military counterpart? First, if no one from the civilian osteopathic medicine sector helps the military develop clinical monitors, someone in the military, possibly a nonDO will. These monitors may be based on a knowledge and understanding of osteopathic medicine that may be inaccurate and superfi- cial. Typical examples of such misinformation conveyed by non-DOs are demonstrated, first, in a Reader's Digest book, The Complete Manual of Fitness and Well-Being. ${ }^{3}$ Information presented in the section on "Osteopathy and Chiropractic," is erroneous and potentially damaging, recommending that the reader avoid going to an "osteopath" for a slipped disk. A 1988 article $^{4}$ in the American Cancer Society's journal lumps early osteopathy and chiropractic together and labels them "unorthodox medicine."

Second, one can be assured that in several years this quality assurance review process already in use in the military will be used in evaluating civilian medical practice. Similar clinical monitors may be used to judge OMT and potentially regulate osteopathic medicine. From personal experience, I can attest that clinical monitors have a tendency toward inaccuracy that may limit the scope and use of OMT in medicine.

Already, peer review organizations, like the Texas Medical Foundation, are starting to use a similar process in evaluating outpatient visits for Medicare recipients. ${ }^{5}$ The Texas peer review organization uses this information generated by the review process to determine the overall quality of medical care given by each physician. The data are stored and can be used later in potential disciplinary action, corrective action, et cetera, against physicians not practicing up to the set standards of care. Keep in mind that the quality assurance clinical monitors are a direct result of the standards of care determined by the governing medical authority, which at this time is made up primarily of those in the allopathic community.

Third, from 4 years' experience working in a large bureaucracy, I know that once something is written and published, it is very difficult to change. Therefore, it is important that civilian and military osteopathic physicians become involved so that this issue is addressed correctly the first time.

\section{Purpose of quality assurance program}

Most physicians do not understand the quality assurance process, or clinical monitor, or their purpose. Quality assurance is not taught 
in medical school. Only through the trial-anderror method in postgraduate programs or active practice do physicians get a glimpse of quality assurance/risk management. Physicians entering the military medical system are astonished at an entire system of vocabulary, rules, and regulations that are very difficult for the seasoned physician to understand.

The quality assurance program is a system of patient care and physician review process insuring a basic standard of medical care. Quality assurance involves a review of credentials of all medical personnel and review of a percentage of patient visits. Its purpose, in addition to reviewing credentials, is to identify areas of substandard patient care, liability, and poorly qualified practitioners, and to review current problems to upgrade the overall quality of medical care. The main working component of this system is the quality assurance checklist.

A quality assurance screening checklist, also called a clinical monitor, is a simple list of the basic elements of a condition, that is, disease, diagnosis, presenting symptom, or procedure) from chief complaint to diagnosis and treatment plan. The checklist includes the clinical monitoring criteria, listed in a logical, stepwise fashion, that the physician must document to justify his or her diagnosis and treatment. The reviewer can quickly scan the chart and determine if the evaluation was thorough, the diagnosis accurate, and the treatment plan appropriate for the diagnosis. All procedures are reviewed for proper documentation of indications, contraindications, work-up, and informed consent. The individual items that can be answered in a yes/no/not-applicable format are called clinical monitoring criteria.

The screening checklist is set up so that if the record is written properly, then $100 \%$ compliance is reported for that chart. Charts are selected on a random basis or by diagnosis. If a physician's compliance is reported to be less than what was previously determined to be acceptable, that provider is reviewed and monitored on a more frequent basis. This process demonstrates the critical importance of superb documentation and record keeping. Any laxity in charting will invariably invite increased re- cord reviews and potential disciplinary action.

Any patient complaint, less than optimal result, or unnecessary procedure is meticulously reviewed and the results filed. Medical providers identified with problems are counseled, sent to training programs, or have disciplinary action taken against them or are subjected to all three actions. The credential files also reflect this documentation.

Clinical monitors simplify medical care into a cookbook approach. A patient has a group of symptoms, signs, laboratory findings. The physician determines the diagnosis that most closely fits the patient's constellation of findings which in turn determines the type of treatment modalities available. Medical care is judged by this standard of care, and the practice of medicine tends to gear itself toward satisfying the judgment process.

Osteopathic medicine and OMT are difficult to fit into this model. No attention is given to the patient and his or her ability to fight disease and remain healthy. For example, with a chief complaint of shoulder pain from minor trauma, a patient may not immediately require manipulation of the glenohumeral joint. Traditional evaluation must categorize the pathologic abnormality, after which the osteopathic physician may choose the best manipulative approach for the patient in a total treatment program. Often, treatment begins at seemingly distant areas first before the shoulder complex is addressed.

Although the purpose of clinical monitoring is not to dwell on the negative side of a physician's practice, it frequently ends up doing so. Healthcare providers frequently have less-than-optimal treatment outcomes and patient complaints. Since no two persons or illnesses are alike, the situation may not fit the clinical monitor. Finally, there is the language limitations of the checklist and the various interpretations by the reviewer of terms used in the list. A built-in margin of error and subjectivity exists that is seldom considered.

One must ask, Who develops these clinical monitors? and Who does the reviewing? First, physicians, preferably experts or physicians of like specialty (or both), are supposed to develop the clinical monitoring criteria on the basis 
of a generally accepted standard of care. In the military, many different personnel, such as physician assistants, registered nurses, senior ranking technicians, and administrators, are involved with physicians in the development and review process. Second, the initial reviewer of physicians, both military and civilian, is not a physician. ${ }^{5}$ A nurse or senior technician is frequently the gatekeeper of the quality assurance cascade. Physicians review any charts selected by the initial screen. They determine if the clinical monitoring criteria have been met satisfactorily. Although these physician reviewers should have a background and specialty similar to that of the physician being reviewed, my personal experience is that this is not always true. Size of the facility and availability of physicians and specialists will determine the physician reviewers. Therefore, the quality of the review process in various facilities may not be equal.

Additionally, one must consider the numerous people - medical and nonmedical - who perform some kind of manipulation, use a handson approach, or work with the musculoskeletal system. Allopathic physicians of various specialties are learning and performing manipulation. Several organizations, such as the North Academy of Musculoskeletal Medicine, and specialists in orthopedic medicine teach skills in manipulation. Physical therapists use all types of manipulation from range of motion to active mobilization. Chiropractors use spinal adjustments and applied kinesiology. Other hands-on therapists include massage therapists and physical therapy assistants.

Osteopathic physicians must differentiate themselves from the others because of the unique philosophy and training of osteopathic medicine. DOs must show where they fit into the medical system, otherwise the government will place OMT with all other manipulations and regulate accordingly. The osteopathic profession must make sure that OMT has a place in medicine when quality assurance monitoring appears in the civilian sector. The screening checklists must be specific enough to serve their purpose, yet general enough to span the scope of osteopathic philosophy and techniques as taught in the colleges of osteopathic medicine

\section{Application of quality assurance to OMT}

It is imperative that clinical monitors are developed specifically for OMT. Separate clinical monitors need to be developed for the other types of manipulation, such as active mobilization (physical therapy), spinal adjustments (chiropractic), and massage therapy. The philosophy, scope, and purpose of the various types of manipulation differ with each discipline. Because surgeons develop and review clinical monitors in surgical cases, osteopathic physicians must do the same in cases involving OMT.

Another important fact that must be emphasized constantly is that osteopathic manipulation is a procedure, meaning that it is treatment performed by skilled physicians and not therapy performed by therapists. The osteopathic physician needs to be credentialed to perform OMT just as any physician needs to document proficiency, knowledge, and skill in any procedure in the credentialing process.

\section{Credentials}

Disagreement and lack of understanding in documenting adequate credentials and expertise to perform OMT in the Navy and an even larger discrepancy in documenting quality care and ongoing clinical monitoring in all aspects of military medicine are evident from the literature $^{2}$ and from the letter from J. Klos, DO (Feb 27, 1988). Presently, most military facilities recognize that graduates of accredited colleges of osteopathic medicine meet the basic diagnostic and skill requirements for performing OMT. For those facilities that do not, documentation of training in OMT can be determined by obtaining transcripts from the physician's college of osteopathic medicine.

The hours spent in class and laboratory can easily be calculated. For example, Kirksville College of Osteopathic Medicine has 267 hours of classroom and laboratory time (plus 20 hours of electives) in the first 3 years, then additional hours are accumulated in the gerontology program, clinical rotations, internships, and residencies. The physician's further training and expertise in OMT can be documented by completion of a residency in osteopathic manipulative medicine or certification 


\section{Quality Assurance Screening Checklist Osteopathic Manipulative Treatment (OMT)}

I. Diagnosis*

A. Somatic dysfunction (list area[s])

B. Other diagnoses - List:

1. Structural component of above diagnosis (A or B) Examples: Neuromusculoskeletal, lymphatic, autonomic, vascular, arthrodial. All are indications for OMT?

*A or B is adequate for quality assurance purposes.

II. Type of technique used and documented (one is adequate for quality assurance purposes)

A. Soft tissue: Stretch, knead, range of motion, myofascial, lymphatic pump

B. Direct: High velocity/low amplitude (HVLA) or thrusting, muscle energy, articulatory

C. Indirect: Myofascial, Jones counterstrain, indirect with respiratory forces, craniosacral

III. Performed by a graduate of a college of osteopathic medicine

IV. Specific contraindications

A. With thrusting-type technique (HVLA) only

1. Vascular insufficiency cervical spine

(thrombi, arteriosclerosis)

2. Severe arthritis of cervical spine (especially rheumatoid arthritis)

3. Acute cervical sprain/strain (whiplash)

4. Acute fracture (traumatic or pathologic

5. Severe and brittle osteoporosis

6. Cancer metastatic to bone

7. Congenital anomaly of vertebral column creating potential cord compression

8. Acute herniated nucleus pulposus within 72 hours of onset (manipulation of that segment)

9. Acute spondylitis within 72 hours of onset

10. Spinal cord tumor

11. Acute onset of dizziness/vertigo without work-up

12. Anticoagulation therapy/bleeding disorder

13. Osteomyelitis, disk infection or abscess (manipulation of that segment)

14. Ocular lens implant, retinal detachment/tear

15. Acute cerebrovascular accident

Total criteria applicable

Total criteria achieved

Percent compliance

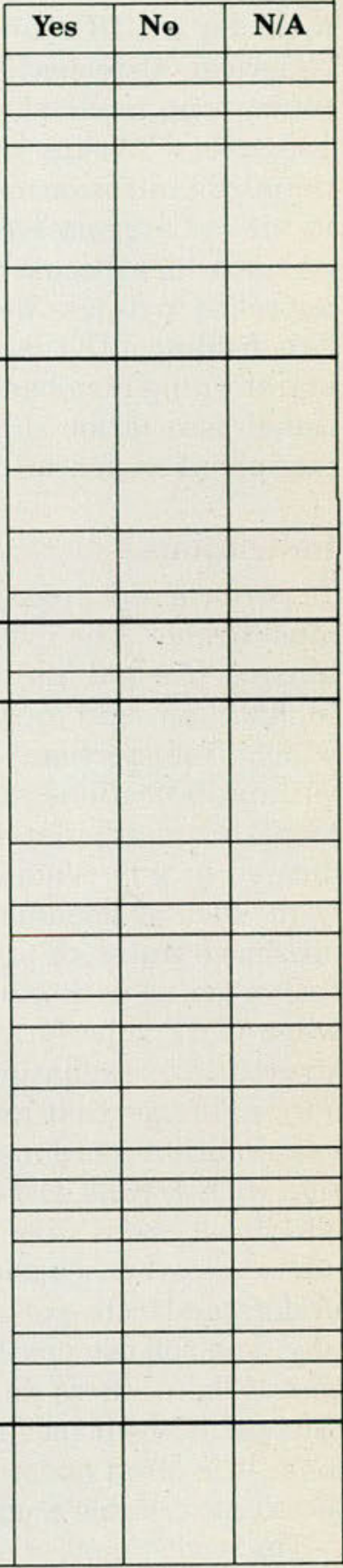

Figure. 
in special proficiency in osteopathic manipulative medicine.

Documentation that a basic level of skill in OMT is maintained is indicated by the requirement for all DOs to have a minimum of 60 American Osteopathic Association--approved continuing medical education hours (out of 150) every 3 years to maintain state and national organization memberships. ${ }^{6}$ These credits are in Category 1-A formal educational programs, which discuss the osteopathic medicine approach in illness, disease, and injury. Therefore, holding a DO degree and state license and maintaining membership in the state and national association should satisfy any misgivings about credentialing DOs to perform OMT.

\section{Indications}

To perform any procedure, certain indications must be met. The indications for OMT are extensive. It is not limited to trauma, whiplash, cephalalgia, and musculoskeletal conditions, which, from personal experience, are the prevailing indications in military medicine. Osteopathic manipulative treatment may be indicated in any condition that affects the patient, such as pneumonia, hypertension, postoperative states, or psychiatric illnesses. Confusion arises with other healthcare providers when OMT is performed after an osteopathic structural examination, especially if the chart only indicates that an osteopathic structural examination was done, according to conversation with J. Klos, DO (March 31, 1988). Much education about this is needed, because most nonosteopathic healthcare professionals do not understand osteopathic vocabulary, philosophy, and the osteopathic structural examination. When others do not understand where osteopathic medicine fits into mainstream medicine, it is often questioned, disputed, and rejected as a viable modality.

The basic tenets of osteopathic medical philosophy are as follows: ${ }^{7}$

- The body is a functional unit. This holistic view states that one area of the body cannot be healthy or ill without influencing the rest of the body.

- The body has self-regulating mechanisms that produce natural and acquired immu- nity, regulate vital functions by homeostasis, repair tissue damage, and compensate for irreparable changes.

- A direct interrelationship exists between structure and function. The neuromusculoskeletal system is the central mechanism by which the organism interacts with its internal and external environment. It is also the means by which an osteopathic physician can influence this structure/function relationship with the use of OMT.

- Osteopathic treatment is based on an understanding and application of the first three principles. The goals of OMT are to eliminate or reduce causative factors, relieve distressing symptoms, compensate for abnormal function, and help the body resist injury and recover from it. ${ }^{7}$

Osteopathic manipulative treatment is the chief procedure that allows all body systems to function up to their potential, or to compensate to the best of their ability. This form of treatment facilitates the healing process and helps the patient achieve a state of health. Osteopathic manipulative treatment is concerned with the structural component of a diagnosis. More importantly, it addresses the patient with the diagnosis.

Documentation of a diagnosis of somatic dysfunction is one of the primary indications for the use of OMT. Somatic dysfunction is defined as follows: ${ }^{8}$

Impaired or altered function of related components of the somatic (body framework) system: skeletal, arthrodial, and myofascial structures, and related vascular, lymphatic, and neural elements. The positional and motion aspects of somatic dysfunction may be described by use of three parameters: (1) The position of the element as determined by palpation; (2) The direction in which motion is freer; and (3) The direction in which motion is restricted.

Somatic dysfunction may be acute, chronic, or secondary, but these will not be discussed here. The diagnosis of somatic dysfunction, with its broad-reaching definition, can be applied to the main cause, contributing factors, or component parts of all disease processes. It is best known for its major role in pain syndromes, and in postural and musculoskeletal condi- 
tions, but these are not its only indications.

Osteopathic manipulative treatment may also be indicated if the osteopathic physician wants to address structural components that are involved. These components are found in the somatic system and its "related elements" during the osteopathic structural examination. Therefore, OMT is indicated in any condition where the osteopathic physician (based on his or her training and experience) believes that it will help the patient recover more quickly, promote healing, alleviate symptoms, prevent occurrence or worsening of the condition, maintain the present health status, and/or enable use of a lower dosage of medications.

\section{Diagnosis}

Somatic dysfunction is a coded diagnosis accepted by the federal government and listed in the International Classification of Diseases, 9 th revision. ${ }^{9}$ There should be no doubt that OMT is the procedure of choice when indicated by a diagnosis of somatic dysfunction. The alterations of position and motion are found during an osteopathic structural examination. However, simply documenting that an osteopathic structural examination was done and OMT was performed is not adequate. Osteopathic physicians should document findings in objective terms that are understandable and reproducible by the entire medical profession, such as degrees in range of motion, specific areas of spasm, pain, redness, or swelling. This procedure will give credence to osteopathic medicine, and enhance further communication among all medical disciplines.

\section{Contraindications}

Osteopathic manipulative treatment, like any procedure, involves some risks. There is a paucity of literature discussing the morbidity and mortality associated with OMT. ${ }^{10-12}$ Most authorities ${ }^{13-19}$ believe that morbidity and mortality rates for manipulation are very low. Deyo ${ }^{14}$ has stated:

... less than 50 cases have been described in the literature, making this an extremely rare event, contrasting with the vast number of treatments given annually. The incidence is probably less than 1 per 3 million treatments, and the events occur only with cervical spine manipulation.

Daneshmend and associates ${ }^{10}$ believe there is a slightly higher incidence of harmful injury following cervical manipulation than the figure just quoted. Nonetheless, the incidence is not less than 1 in 1 to 2 million cases at a very conservative estimate. One article has documented a few cases of injury after manipudlation of the lumbar spine. ${ }^{12}$ However, the vast majority of cases reported involved the cervical spine, and always were caused by a thrusting maneuver.

When the incidence of risks of OMT is compared with the incidence of serious side effects of other procedures or of medications, OMT is relatively innocuous. About $5 \%$ of admissions to hospitals are the result of adverse reactions to drugs. ${ }^{20}$ In 1981 , Steel and coworkers ${ }^{21}$ reported that $36 \%$ of patients on an internal medicine service had some adverse response to a drug or to a diagnostic or therapeutic procedure. Of these reactions, $9 \%$ either were lifethreatening or resulted in serious disability.

Absolute contraindications for manipulation are relatively few. These would be apparent as the osteopathic physician goes through a common-sense screening process in the workup and treatment of a patient. Although OMT is not contraindicated in any case, certain manipulative techniques may be contraindicated because of the patient's condition and the forces involved in the technique; for example, high velocity/low amplitude (HVLA) or thrusting techniques applied to the cervical spine with such conditions as acute fracture, cancer metastatic to bone, or severe osteoporosis (Figure). It is with such conditions that the majority of complications occur. This is not to say that all techniques in the cervical spine are contraindicated: Soft tissue and indirect techniques may be applied and are effective.

There are a few relative contraindications, which are general in nature and are based on the physician's expertise and the patient's condition. Milder forms of arthritis, chronic disk disease, osteoporosis, peripheral vascular disease, chronic disease states, and hypermobile joints are examples of conditions for which the osteopathic physician may want to use less 
forceful techniques. Generally, the more seriously and acutely ill the patient (such as hospital patients), the gentler and briefer the treatment. Also, only the acute somatic dysfunctions relating to a serious illness are addressed in an OMT. The chronic somatic dysfunctions are usually left for outpatient care unless they are a major contributor to the disease process.

\section{Technique}

Choice of manipulative technique is based on the patient's condition and the osteopathic physician's skill. Three types of techniques are listed in the clinical monitor checklist:

1. Soft tissue techniques consist of stretching, kneading, effleurage, range of motion (active and passive), myofascial stretch and release, and lymphatic pump.

2. Direct techniques consist of positioning the specific tissue or joint against its restrictive barrier and mobilizing the restriction. Such techniques include HVLA thrust, muscle energy (where muscle contractions can be used to remove the restriction), or range of motion (articulatory forces).

3 . Indirect techniques involve taking the specific tissue or joint away from the restrictive barrier and using respiration to allow the body's inherent forces to correct the dysfunction. Specific forms of indirect technique include indirect, counterstrain, myofascial release, and craniosacral techniques.

The quality assurance screening checklist (Figure) is a suggested protocol that could be used by the military in evaluating the proper use of OMT by the osteopathic physician. The checklist provides sufficient detail to fulfill its purpose but is not so detailed that its use would be limited.

This paper has addressed an issue that may involve the whole osteopathic profession: quality assurance/risk management in the use of OMT. Information about the military quality assurance review system may help civilian physicians to understand the process and the consequences of its spread to civilian practices.
Application of osteopathic principles to quality assurance issues is necessary if appropriate clinical monitors are to be developed for OMT.

1. Mack RA: For DOs, military fulfills promise to be all that you can be.' The DO 1988;29:79-85.

2. Navy rescinds OMT ban policy AMOPS Newsletter 1987;7(September):3.

3. Reader's Digest (eds): The Complete Manual of Fitness and Well-Being. Westminster, Md, Random House Books, 1988, p 312

4. Cassileth BR, Brown H: Unorthodox cancer medicine. $C A$ 1988;38:176-186.

5. Peters RL: Medical peer review as performed by the Texas Medical Foundation. J Osteopath Med 1988;2:37-40.

6. Reuther GA: AOA continuing medical education. JAOA 1989;89:1456-1460.

7. Kirksville College of Osteopathic Medicine and Surgery: The osteopathic concept-an interpretation. Reprinted from The Journal of Osteopathy October 1953 and April through October 1954.

8. 1989-1990 Yearbook and Directory of Osteopathic Physicians. Chicago, American Osteopathic Association, 1989.

9. International Classification of Diseases, 9 th revision: Clinical Modification, ed 2. Us Department of Health and Human Services (PHS) 80-1260. US Government Printing Office, 1980, vol $2, \mathrm{p} 2$.

10. Daneshmend TK, Hewer RL, Bradshaw JR: Acute brain stem stroke during neck manipulation. Br Med J 1984;288:189.

11. Laughlin TM: Complications of spinal manipulation. Osteopath Ann 1984;14:21-3.

12. Dan NG, Saccasan PA: Serious complications of lumbar spinal manipulation. Med J Australia 1983;2:672-673.

13. Goldstein M (ed): The Research of Spinal Manipulative Therapy. National Institute of Neurological and Communicative Disorders and Stroke Monograph No. 15. DHEW publication No (NIH) 76-498. National Institutes of Health, 1975.

14. Curtis P: Spinal manipulation. State Art Rev Occup Med 1987;2:31-44.

15. Maigne R: Orthopedic Medicine: A New Approach to Vertebral Manipulations, translated and edited by WT Liberson. Springfield, Ill, Charles C Thomas, 1972, pp 165-172.

16. Kirkaldy-Willis WH (ed): Managing Low Back Pain. New York, Churchill Livingstone, 1983, chapter 13.

17. Krueger BR, Okazaki H: Vertebral-basilar distribution infarction following chiropractic cervical manipulation. Mayo Clin Proc 1980;55:322-332.

18. Grieve GP (ed): Modern Manual Therapy of the Vertebral Column. New York, Churchill Livingstone, 1986, pp 873-884.

19. Maitland GD: Vertebral Manipulation, ed 5. Sevenooks, Kent, England, Butterworth, 1986, chapter 2.

20. Rodman GP, Schumacher HR (eds): Primer on Rheumatic Diseases, ed 8. Atlanta, Arthritis Foundation, 1983, p 81.

21. Steel K, Gertman PM, Crescenzi, et al: Iatrogenic illness on a general medical service at a university hospital. $N$ Engl $J$ Med 1981;304:638-642. 